\title{
Caracterización química, degradabilidad ruminal y aceptabilidad por bovinos, de un subproducto de la industria del papel
}

\section{Chemical characterization, ruminal degradability and bovine acceptability of a byproduct of the paper industry}

\author{
George Oneiber Jaime ${ }^{1 *}$; Juan Evangelista Carulla ${ }^{2}$; Gustavo Álvaro Wills-Franco ${ }^{3}$
}

\begin{abstract}
'Zootecnista, M.Sc. Universidad Nacional de Colombia, Facultad de Medicina Veterinaria y de Zootecnia. Bogotá, Colombia; e-mail: gojaimet@unal.edu.co; (iD) https://orcid.org/0000-0003-0020-978X

ZZootecnista, M.Sc., Ph.D. Universidad Nacional de Colombia, Facultad de Medicina Veterinaria y de Zootecnia. Bogotá, Colombia; e-mail: jecarullaf@unal.edu.co; (D) https://orcid.org/0000-0002-8854-1850

${ }^{3}$ Zootecnista, M.Sc. Universidad Nacional de Colombia, Facultad de Medicina Veterinaria y de Zootecnia. Bogotá, Colombia; e-mail: gawillsf@unal.edu.co; (D) https://orcid.org/0000-0003-1088-0817

*autor de correspondencia: gojaimet@unal.edu.co
\end{abstract}

Cómo citar: Jaime, G.O.; Carulla, J.E.; Wills-Franco, G.A. 2021. Caracterización química, degradabilidad ruminal y aceptabilidad por bovinos, de un subproducto de la industria del papel. Rev. U.D.C.A Act. \& Div. Cient. 24(1):e1204. http://doi.org/10.31910/rudca.v24. n1.2021.1204

Artículo de acceso abierto publicado por Revista U.D.C.A Actualidad \& Divulgación Científica, bajo una Licencia Creative Commons CC BY-NC 4.0

Publicación oficial de la Universidad de Ciencias Aplicadas y Ambientales U.D.C.A, Institución de Educación Superior Acreditada de Alta Calidad por el Ministerio de Educación Nacional.

Recibido: abril 22 de $2019 \quad$ Aceptado: abril 19 de $2021 \quad$ Editado por: Ingeborg Zenner de Polanía

\section{RESUMEN}

Las variaciones climáticas pueden limitar la disponibilidad y la calidad de los forrajes. Se estudió un subproducto del papel (SIP) como alternativa a los forrajes en la ración de los bovinos. Se analizaron 12 muestras de SIP, para determinar su composición nutricional y degradabilidad ruminal. La aceptabilidad (1,07kg MS) por los bovinos de los SIP solo o en mezcla, se evaluó en 12 novillas Holstein, asignadas a dos cuadrados latinos, cada uno con tres tratamientos y dos réplicas. En el primer cuadrado, los tratamientos fueron SIP solo (T1); SIP+glicerol (T2) y SIP+melaza (T3). En el segundo, los tratamientos fueron SIP+suplemento comercial (SC) (T4); SIP+SC+glicerol (T5) y SIP+SC+melaza (T6). Los SIP presentaron bajos contenidos de PC $(1,5 \pm 0,14 \%)$ y altos niveles de FDN $(56,9 \pm 6,17 \%)$ y cenizas $(53,3 \pm 1,98 \%)$ y una $D I V M O$ $(47,7 \pm 5,8 \%)$ media. Las concentraciones de $\mathrm{P}(0,045 \pm 0,006 \%)$ y $\mathrm{K}$
$(0,023 \pm 0,03 \%)$ fueron bajas, mientras que las de $\mathrm{Ca}(13,5 \pm 0,8 \%)$, $\mathrm{Cu}(63,9 \pm 11,5 \%)$ y $\mathrm{Fe}(1365 \pm 189 \mathrm{ppm})$ fueron altas. La tasa de desaparición de la MS del SIP fue de $11,8 \% * \mathrm{~h}^{-1}$; la fracción soluble (a), 14\% y la fracción potencialmente degradable y no soluble (b), 46,6\%. La aceptabilidad del SIP fue mayor, cuando se ofreció en mezclas (T1 vs T2-T3; p<0,05) y con concentrado y melaza (T4-T5 vs T6; $\mathrm{p}<0,05)$. La variación en la calidad composicional del SIP fue menor para la mayoría de nutrientes y su calidad baja, debido a una digestibilidad media, una baja PC y altas cenizas. Además, su aceptabilidad fue pobre cuando se suministró sola, pero mejoró con el uso de palatabilizantes o SC.

Palabras clave: Aceptabilidad; Digestibilidad in vitro; Degradabilidad ruminal; Subproductos; Papel. AGROVOC Tesauro. 


\section{ABSTRACT}

Climatic variations can limit the availability and quality forages. The use of byproducts of the paper industry (BPI) was studied as part of cattle rations. Twelve samples of BPI were analyzed to determine the nutritional composition and ruminal degradability. The acceptability (1.07 kg DM) of the BPI was evaluated in 12 Holstein heifers, assigned to two Latin squares with two replicates and three treatments each. The first square, the treatments were BPI alone (T1), $\mathrm{BPI}+$ glycerol (T2) and BPI + molasses (T3). In the second square, treatments were BPI + commercial supplement (CS) (T4), BPI +CS + glycerol (T5) and BPI + CS + molasses (T6). The BPI presented low CP $(1.5 \pm 0.14 \%)$, high levels of NDF $(59.6 \pm 6.17 \%)$ and ash $(53.3 \pm 1.98 \%)$ and médium IVODM (47.7 $\pm 5.8 \%)$. Concentrations of $\mathrm{P}(0.045 \pm 0.06 \%)$ and $\mathrm{K}(0.023 \pm 0.03 \%)$ were low while $\mathrm{Ca}$ $(13.5 \pm 0.8 \%), \mathrm{Cu}(63.9 \pm 11.5 \mathrm{ppm})$ and $\mathrm{Fe}(1365 \pm 189 \mathrm{ppm})$ high. Rate of DM disappearance for BPI was $11.8 \% * \mathrm{~h}^{-1}$, soluble fraction (a) $14 \%$ and a potentially degradable fraction but not soluble (b) $46.6 \%$. The acceptability of BPI was higher when fed in mix (T1 vs T2-T3; $<<0.05$ ) and with concentrate and molasses (T4-T5 vs T6; $\mathrm{p}<0.05)$. Variation in nutritional composition of BPI was low for most and its nutritional quality low due to a moderate digestibility, a low CP and high ash. In addition, its acceptability was low but improved when supplied with the use of palatability agents and CS.

Keywords: Acceptability; in vitro digestibility; Ruminal degradability; Byproduct; Paper.

\section{INTRODUCCIÓN}

El aumento de la actividad agroindustrial en los últimos años, ha tenido como consecuencia la acumulación de residuos lignocelulósicos en el mundo (Mahesh \& Mohini, 2013), generados por prácticas forestales y agrícolas, industrias madereras, productoras de pulpa y papel, entre otras, los cuales son, a menudo, eliminados mediante la combustión de biomasa, que genera contaminación ambiental (Howard et al. 2003). Algunos residuos sólidos derivados de la industria de la pulpa y papel han tenido diferentes opciones de aprovechamiento, entre las cuales, están incineración, compostaje, vermicompostaje, producción de etanol, producción de ácido láctico, uso en la industria papel y cartón, productos minerales (cemento, cerámicas), pavimentos, productos absorbentes y alimentación animal (Bajpai, 2015). El uso de algunos tipos de residuos de la industria del papel en la alimentación de animales rumiantes se ha evaluado desde los años setenta, en donde autores, como Mertens et al. (1971) y Coombe \& Briggs (1974), evaluaron la inclusión de papel en la dieta de bovinos y de ovinos y otros autores, como Croy \& Rode (1988), Moss et al. (1990), Nishimura et al. (2019), Maeda et al. (2019) e Izumi et al. (2019), evaluaron la inclusión de pulpa de papel en la dieta de rumiantes.

Otros residuos generados durante el proceso de fabricación de papel, como los lodos de destintado (Subproductos de la Industria del Papel -SIP), no han sido evaluados en alimentación animal. Estos lodos de destintado o SIP, se generan en la producción de fibra, a partir de papel reciclado (Kujala, 2012); sus componentes principales son fibras cortas de celulosa, carbonato de calcio, caolín, minerales y algunas partículas de tinta (Monte et al. 2009).

En Colombia, la producción de papel y cartón, para el 2017, fue 1.252.680 toneladas, donde el 60,81\% de la fibra utilizada tuvo su origen a partir de fibra secundaria (papel reciclado) y se generaron alrededor de 611.245 toneladas de residuos no peligrosos (ANDI, 2019). La cantidad de SIP generados en la producción de papel, varía del 20 al 40\%, dependiendo del tipo de papel producido (papel periódico o papel tissue) (Kujala, 2012). La empresa que suministró los SIP para esta investigación genera, aproximadamente, 200 toneladas diarias de este material (Productos Familia SAS, comunicación personal).

De acuerdo con los decretos 4741 de 2005 (Ministerio de Ambiente Vivienda y Desarrollo Territorial, 2005) y 1287 de 2014 (Ministerio de Vivienda Ciudad y Territorio, 2014), los SIP se catalogan como "residuos industriales no peligrosos", ya que para las características, como corrosividad $(\mathrm{pH})$, toxicidad (contenidos $\mathrm{mg} / \mathrm{L}$ de As, Ba, $\mathrm{Cd}, \mathrm{Cr}, \mathrm{Hg}, \mathrm{Ag}, \mathrm{Pb}$ y Se), metales totales y microbiológicas (fagos somáticos, Sallmonella sp. y huevos de helmintos), los SIP cumplen con los valores establecidos en estos decretos.

El uso de subproductos agroindustriales fibrosos puede constituir una fuente suplementaria de alimento para los rumiantes en épocas de baja oferta forrajera (Villas-Bôas et al. 2002). Esta especie animal, por medio de su sistema digestivo, tiene la capacidad de transformar materiales fibrosos en productos de alta calidad proteica, como son la leche y la carne (Gómez et al. 2014).

Los objetivos de este trabajo fueron: 1) Caracterizar químicamente los SIP y determinar su variabilidad en muestras provenientes de diferentes lotes de producción y evaluar la degradabilidad ruminal (MS y FDA) y 2) Determinar la aceptación de los SIP en bovinos, usando glicerol y melaza, como productos palatabilizantes.

\section{MATERIALES Y MÉTODOS}

Ubicación temporal y espacial de la prueba. Los análisis químicos, digestibilidad in vitro de la materia seca y orgánica (DIV MS, DIVMO) y degradabilidad in situ de los SIP, se realizaron en el laboratorio de Nutrición Animal y Facultad de Medicina Veterinaria y de Zootecnia de la Universidad Nacional de Colombia, sede Bogotá, entre agosto y septiembre de 2017.

Caracterización química. Para la caracterización química de las muestras de SIP (lodos de destintado), se analizaron 12 muestras (muestras compuestas del lote de producción de 500gr/cada una), provenientes de diferentes días (lotes) de producción, de una empresa productora de papel (Productos Familia Cajicá S.A.S); los análisis realizados fueron: Materia seca (MS), Proteína cruda (PC), Lignina, Cenizas, Minerales: $\mathrm{Ca}, \mathrm{Cu}, \mathrm{Zn}, \mathrm{Mg}, \mathrm{Fe}, \mathrm{K}, \mathrm{Na}, \mathrm{Mn}$ (espectrofotometría de absorción atómica), P (colorimetría) (AOAC, 2006), fibra detergente neutro (FDN), fibra detergente ácido (FDA) (Van Soest et al. 1991), digestibilidad in vitro de la materia seca (DIVMS) y digestibilidad in vitro de la materia orgánica (DIVMO) 
(Tilley \& Terry, 1963).

Cinética de degradación ruminal. Para la degradabilidad in situ de la MS (DISMS) y FDA, se utilizó un bovino de la raza normando (peso $700 \mathrm{~kg}$ ), provisto de cánula ruminal permanente. El animal, se encontraba pastoreando Kikuyo (Cencbrus clandestinus), con suministro de agua a voluntad. Se siguió la técnica de bolsa de nylon (Ørskov, 2000), con 7 tiempos de incubación en el rumen: $0,3,6,12,24,36,48$ horas (3 repeticiones por cada tiempo de incubación). En cada bolsa, se depositaron $3 g$ de muestra de SIP (se colocó el material seco entero, con un tamaño de partícula de $0,5 \mathrm{~cm}$ de diámetro, aproximadamente, ya que, por su alto contenido de minerales, se presentaron pérdidas de material, a través de la bolsa, cuando se molió), obteniendo una relación de $15 \mathrm{mg}$ de muestra, por cada $\mathrm{cm}^{2}$ de bolsa (Nocek, 1988). Las muestras, se introdujeron de manera inversa, de acuerdo con el tiempo de incubación para ser retiradas al mismo tiempo. Las muestras del tiempo cero (0) fueron sumergidas y retiradas inmediatamente del rumen. Luego del periodo de incubación, las muestras fueron extraídas del rumen, se lavaron con agua corriente y se sometieron a secado, en una estufa de aire forzado, a $65^{\circ} \mathrm{C} / 48 \mathrm{~h}$ y luego se pesaron, para expresar los resultados, como DISMS. Para reportar la degradabilidad in situ de FDA, a los residuos de la degradación in situ se les realizó una extracción con detergente ácido $\left(100^{\circ} \mathrm{C} / 1 \mathrm{~h}\right)$, usando un equipo analizador de fibra Ankom® 200 (ANKOM Technology Corp., Nueva York, EUA) (Van Soest, 1994). Los parámetros de la cinética de degradabilidad ruminal de los SIP, se estimaron para MS y FDA usando el modelo de Ørskov \& McDonald (1979) y la degradabilidad efectiva (DE) fue determinada usando la ecuación propuesta por Bhargava \& Ørskov (1987).

Prueba de aceptación. La prueba de aceptación (consumo) de los SIP por los bovinos, se llevó a cabo entre mayo y junio de 2017, en el municipio de Mosquera (Cundinamarca, Colombia), a una altitud $2.650 \mathrm{~m}$ s.n.m., temperatura promedio anual de $13^{\circ} \mathrm{C}$, humedad relativa de 80 a $85 \%$. Este experimento tuvo una duración total de 30 días, divididos en 3 periodos (10 días cada periodo), en los cuales, 7 días correspondieron a la fase de acostumbramiento a los tratamientos y 3 días, de evaluación de consumo. Para cada periodo, se registró la oferta y el rechazo de cada uno de los tratamientos y se tomó, como valor del consumo, el promedio de los tres días de evaluación (días 8, 9 y 10). Se utilizaron 12 novillas Holstein (23,8 +/- 3,6 meses y $407+/-49 \mathrm{~kg}$ de peso), que se asignaron en 2 cuadrados latinos replicados, con 3 tratamientos a evaluar en cada uno (Tabla 1), con una distribución (3X3X3X2), que corresponde a los periodos, tratamientos, animales y cuadrados, respectivamente (Martínez et al. 2011).

La cantidad de SIP ofrecidos a los animales (1,07kg de MS/animal/ día), correspondieron al 10\% del consumo total de MS estimada (10,7kg de MS/animal/día, según NRC, 2001); el glicerol y la melaza adicionada (0,221 y $0,208 \mathrm{~kg}$ de MS, respectivamente), al $20 \%$ del total de los SIP ofrecidos y el suplemento comercial $0,89 \mathrm{~kg}$ de MS/ animal/día (PC 16\%, Grasa 2,5\%, Fibra cruda 15\%, Ceniza 10\%). La cantidad de SIP suministrada, se determinó por la restricción que suponía la concentración de $\mathrm{Cu}$ en este material y los palatabilizantes por pruebas de campo, donde la cantidad del $20 \%$ estimuló el consumo por los animales. Las novillas, se encontraban pastoreando una pradera de Kikuyo (C. clandestinus), bajo el sistema de franjas. Los animales, se recogían y eran llevados a un establo (7:00a.m.), a un puesto individual, donde se les suministró, en un comedero, el respectivo tratamiento; adicionalmente, a cada animal, se le ofreció $80 \mathrm{~g} /$ día de sal mineralizada en el potrero.

Análisis estadístico. Para los datos de composición química, DIVMS DIVMO de los SIP, se hizo un análisis de estadística descriptiva.

Para la prueba de aceptabilidad de los SIP, los datos de cada cuadrado latino, se sometieron a un análisis de varianza para un diseño de cuadrado latino replicado ( 3 X 3 X 3 X 2), usando la función PROC GLM de SAS (Sistema de Análisis Estadístico), de acuerdo con el siguiente modelo: $Y_{i j(k)}=\mu+Y_{l}+\alpha(Y)_{i l}+\beta(Y)_{j i}+\tilde{i}_{(k)}+\epsilon_{i j(k)}$, donde:

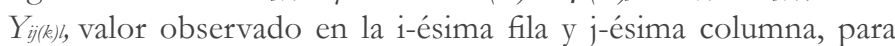
el tratamiento k-ésimo del l-ésimo cuadrado, $\mu=$ media general, $\mathrm{Y}_{1}=$ efecto del 1 -ésimo cuadrado $(1=1,2), \boldsymbol{\alpha}(\mathrm{Y})_{\mathrm{il}}=$ efecto de la

Tabla 1. Materia seca ofrecida y composición de los tratamientos (Cuadrado latino 1 y 2).

\begin{tabular}{|c|c|c|c|c|c|c|}
\hline \multirow[t]{2}{*}{$\begin{array}{c}\text { Cuadrado } \\
\text { Latino }\end{array}$} & \multirow[t]{2}{*}{$\begin{array}{c}\text { No. de } \\
\text { animales }\end{array}$} & Tratamiento & SIP & $\begin{array}{c}\text { Suplemento } \\
\text { comercial }\end{array}$ & Glicerol & Melaza \\
\hline & & & \multicolumn{4}{|c|}{ kg de MS } \\
\hline \multirow{3}{*}{1} & \multirow{3}{*}{6} & T1 & 1,07 & -- & --- & -- \\
\hline & & $\mathrm{T} 2$ & 1,07 & --- & 0,221 & --- \\
\hline & & T3 & 1,07 & --- & --- & 0,208 \\
\hline \multirow{3}{*}{2} & \multirow{3}{*}{6} & $\mathrm{~T} 4$ & 1,07 & 0,89 & --- & --- \\
\hline & & T5 & 1,07 & 0,89 & 0,221 & -- \\
\hline & & T6 & 1,07 & 0,89 & --- & 0,208 \\
\hline
\end{tabular}

SIP = Subproducto de la Industria del Papel. 
i-ésima fila $(\mathrm{i}=1,2,3)$ dentro del l-ésimo cuadrado, $\beta(Y)_{\mathrm{j} 1}=$ efecto de la j-ésima columna $(j=1,2,3)$, dentro del l-ésimo cuadrado, $\tilde{\mathbf{l}}_{(\mathrm{k})}=$ efecto del k-ésimo tratamiento $(\mathrm{k}=1,2,3)$ y $\epsilon_{i j(k) l}=$ error aleatorio. En el modelo, los animales fueron las columnas y los periodos las filas. Para los efectos principales, las diferencias significativas fueron consideradas $(\mathrm{p}<0,05)$. Se realizaron comparaciones de medias no planeadas (prueba de Tukey).

De acuerdo con el acta 06 del 2016, el Comité de Bioética de la Facultad de Medicina Veterinaria y de Zootecnia de la Universidad Nacional de Colombia, sede Bogotá, se avalaron los procedimientos del presente trabajo.

\section{RESULTADOS Y DISCUSIÓN}

Caracterización química. En las muestras analizadas de los SIP (12), se encontró homogeneidad para parámetros, como MS, DIVMS, lignina y cenizas; las mayores variaciones, se encontraron para los contenidos de FDA (48,3\% \pm 6,93), FDN (59,6\% \pm 6,17), DIVMO $(47,7 \% \pm 5,8), \mathrm{Fe}(1365 \mathrm{ppm} \pm 189), \mathrm{Zn}(69,1 \mathrm{ppm} \pm 16,1)$ y $\mathrm{Cu}$ $(63,9$ ppm $\pm 11,5)$ (promedio \pm desviación estándar) (Tablas 2 y 3).

Los SIP son un recurso húmedo, alto en fibras y cenizas y bajo en proteína cruda, lo que coincide con reportes de Bajpai (2015) y Monte et al. (2009), quienes analizaron lodos residuales de la fabricación de papel y Moss et al. (1990), cuando evaluaron pulpa de papel. La digestibilidad in vitro de la MS y MO encontradas en las muestras de SIP, en este trabajo, fueron medias y superiores a las encontradas por Moss et al. (1990), para pulpa de papel reciclado y Coombe \& Briggs (1974), para papel periódico.

Los contenidos de FDN, FDA y lignina de las muestras de SIP, en este estudio, presentan valores inferiores a los reportados por Croy \& Rode (1988), Coombe \& Briggs (1974) y Moss et al. (1990), en diferentes muestras de residuos de pulpa de papel, mientras que Cavka et al. (2013), reportaron contenidos de lignina similares para lodos de fibras de papel. Los valores de FDN sumados a las cenizas superan el $100 \%$ en las muestras de los SIP analizadas en este estudio, debido a la alta concentración de minerales en el producto y que pueden quedar en el residuo del detergente neutro, incrementado los valores de FDN (Van Soest, 1994), por lo cual, se realizó un ajuste de los valores de FDN por el contenido de cenizas de las muestras de los SIP (cFDN) (Tabla 2).

Los contenidos de cenizas de las muestras de SIP fueron altos $(53 \% \pm 1,9)$, consistente con lo descrito por Croy \& Rode (1988) y Méndez et al. (2009), quienes reportan altos contenidos de cenizas en los residuos de la producción de papel. La composición mineral de las 12 muestras de SIP presentó altos niveles de $\mathrm{Ca}$, niveles

Tabla 2. Análisis químico, DIVMS y DIVMO de SIP de 12 días de producción.

\begin{tabular}{|l|c|c|c|c|c|c|c|c|c|}
\hline & MS & PC & $\begin{array}{c}\text { DI- } \\
\text { VMS }\end{array}$ & DIVMO & FDN & FDNc & FDA & Lignina & Cenizas \\
\cline { 2 - 10 } & \multicolumn{8}{|c|}{$\%$} \\
\hline Promedio & 53,7 & 1,5 & 68,4 & 47,7 & 59,6 & 36,9 & 48,3 & 4,9 & 53,3 \\
\hline Mínimo & 50,7 & 1,3 & 63,6 & 33,7 & 47,6 & 33,4 & 35,2 & 3,4 & 50,8 \\
\hline Máximo & 57,7 & 1,7 & 75,1 & 58,4 & 69,6 & 39,5 & 57,6 & 5,7 & 57,9 \\
\hline DS & 2,19 & 0,14 & 3,14 & 5,8 & 6,17 & 1,90 & 6,93 & 0,72 & 1,98 \\
\hline EEM & 0,63 & 0,04 & 0,09 & 1,69 & 1,78 & 0,55 & 2,00 & 0,2 & 0,57 \\
\hline
\end{tabular}

FDNc $=$ Fibra en Detergente Neutro corregido por cenizas; DS = Desviación estándar. $\mathrm{EEM}=$ Error estándar de la media.

Tabla 3. Composición mineral de SIP de 12 días de producción.

\begin{tabular}{|c|c|c|c|c|c|c|c|c|c|}
\hline \multirow{3}{*}{ Promedio } & $\mathrm{Ca}$ & $\mathbf{P}$ & $\mathbf{K}$ & $\mathrm{Mg}$ & $\mathrm{Na}$ & $\mathrm{Cu}$ & $\mathrm{Zn}$ & $\mathrm{Fe}$ & $\mathrm{Mn}$ \\
\hline & \multicolumn{4}{|c|}{$\%$} & \multicolumn{5}{|c|}{ Ppm } \\
\hline & 13,5 & 0,045 & 0,023 & 0,25 & 0,08 & 63,9 & 69,1 & 1365 & 50,9 \\
\hline Mínimo & 12,5 & 0,039 & 0,018 & 0,23 & 0,07 & 48,4 & 52,5 & 1144 & 45,4 \\
\hline Máximo & 14,8 & 0,061 & 0,029 & 0,27 & 0,09 & 89,4 & 113,8 & 1898 & 64,3 \\
\hline DS & 0,8 & 0,006 & 0,003 & 0,014 & 0,005 & 11,5 & 16,1 & 189 & 5,3 \\
\hline EEM & 0,21 & 0,001 & 0,0009 & 0,0041 & 1,53 & 3,33 & 4,66 & 54,58 & 0,001 \\
\hline
\end{tabular}

DS = Desviación estándar; EEM= Error estándar de la media. 
medios de $\mathrm{Mg}, \mathrm{Na}$, Mn y bajos niveles $\mathrm{P}, \mathrm{K}$, al ser comparadas con el contenido de estos minerales en forrajes, como Kikuyo (C. clandestinus) (Bernal, 2003). Minerales, como el Zn, Fe, y Cu, presentan valores elevados, respecto a forrajes, como kikuyo o Raigrás (Bernal, 2003). Se encontró una diferencia entre la cantidad de cenizas presentes en las muestras de los SIP y la suma de los contenidos de los minerales analizados, ya que puede existir una gran cantidad de sílice en este material, debido al uso de arcillas en el procesamiento de este tipo de residuos. El alto contenido de $\mathrm{Cu}$ en los SIP plantea limitaciones en el nivel de incorporación en las raciones de los rumiantes, especialmente en los ovinos, debido a susceptibilidad a altos niveles de este mineral (máximo tolerable $15 \mathrm{mg} / \mathrm{kg}$ de MS) (ARC, 1980), mientras que, para los bovinos, la ingesta recomendada varía entre $10-25 \mathrm{mg} / \mathrm{kg} \mathrm{MS}$, dependiendo de la etapa de lactancia (Goselink \& Jongbloed, 2012).

Cinética de degradación ruminal. La degradabilidad "in situ” de la MS y FDA para las 48 horas de incubación ruminal fue de 62,1\% y $45,5 \%$, respectivamente. La pérdida de MS de los SIP para las bolsas de incubación a la hora cero " 0 ” fue baja $(11,3 \%)$ y se incrementó durante las siguientes 36 horas, donde se estabilizó. La pérdida de FDA de las bolsas en el tiempo cero " 0 " fue $25,9 \%$; posteriormente, se presentó un tiempo "lag" de 6 horas y luego inició su degradación, que se produjo a una mayor tasa en las primeras 24 horas, que en las siguientes 48 horas (Figura 1).

La desaparición de la MS y FDA de los SIP al inicio de la incubación ruminal (tiempo 0) es similar a los valores encontrados por Moss et al. (1990), para residuos de pulpa de papel y por Haile et al. (2017), para diferentes residuos lignocelulósicos; no obstante, la degradabilidad in situ de los SIP fue mayor a las 48 horas de incubación ruminal, respecto a los residuos de la pulpa de papel y residuos de cosecha reportados por estos autores. Croy \& Rode (1988) encontraron una amplia variabilidad en la degradabilidad in sacco de la MS de residuos de pulpa de papel en diferentes tiempos de incubación, dependiendo del origen de la muestra (molino). Espinoza et al. (2017) reportan valores superiores de degradabilidad en el tiempo 0 y 48 horas en residuos agroindustriales de maracuyá y plátano, asociado a los bajos niveles de fibra, de este tipo de residuo. Es deseable que los SIP se combinen con alimentos altamente digestibles, lo cual, estimularía una población microbiana ruminal más activa, se degradaría la fibra más rápido, aumentando la velocidad de paso y el consumo de materia seca por los animales.

Las diferentes fracciones de la MS de los SIP presentaron una baja fracción soluble "a" y una mayor fracción potencialmente degradable "b", una alta velocidad de degradación $\left(k d \% h^{-1}\right)$ y alta degradabilidad efectiva (estimada con una tasa de paso de $5 \% \mathrm{~h}^{-1}$ ), mientras que para el FDA, se encontró una alta fracción "a", baja fracción "b", una baja velocidad de degradación $\left(k d \% h^{-1}\right)$ y baja degradabilidad efectiva (Tabla 4).

La fracción "a" de la MS estimada para los SIP es similar a los reportes de Chumpawadee et al. (2006), Haile et al. (2017) y Bravo et al. (2008), para algunos residuos lignocelulósicos de cosecha y superior a los reportados por Contreras et al. (2019), para residuos de cosecha, como chala de maíz y paja de trigo. El alto valor obtenido

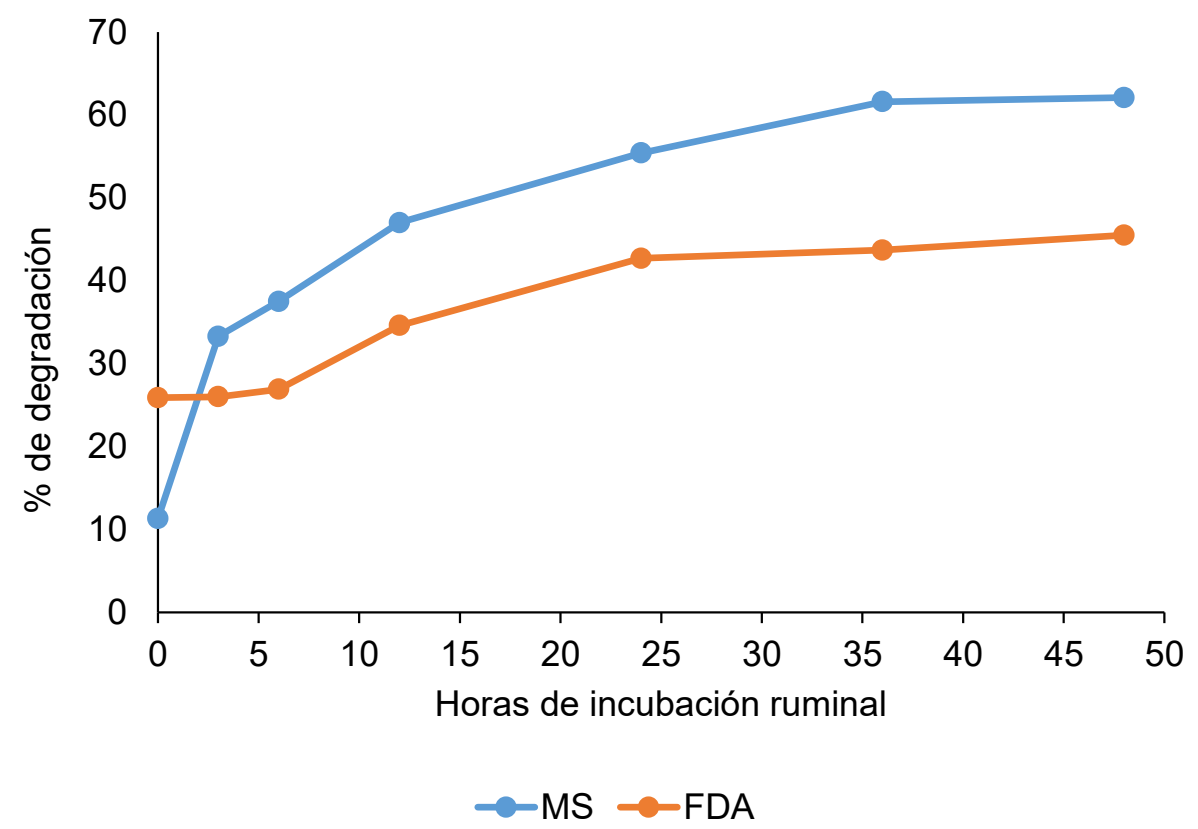

Figura 1. Patrón de degradabilidad "In situ" de la MS y FDA del SIP. 
Tabla 4. Parámetros de la cinética ruminal "In situ” de la MS y FDA de una muestra de SIP.

\begin{tabular}{|l|c|c|c|c|c|}
\hline \multirow{2}{*}{} & \multicolumn{3}{|c|}{ Fracción } & & $\mathrm{DE}$ \\
\cline { 2 - 6 } & $a(\%)$ & $b(\%)$ & $c(\%)$ & $k d\left(\% \mathrm{~h}^{-1}\right)$ & $\left(5 \% \mathrm{~h}^{-1}\right)$ \\
\hline Materia seca & 14,0 & 46,6 & 39,2 & 11,8 & 46,9 \\
\hline FDA & 23,8 & 25,5 & 50,6 & 4,3 & 35,7 \\
\hline
\end{tabular}

$a$ : Fracción soluble; $b$ : Fracción potencialmente degradable; $c$ : Fracción no degradable; kd: Tasa de degradación de la fracción $b$ (\% / hora), DE: Degradabilidad Efectiva.

para la fracción soluble "a" del FDA puede estar relacionado con una pérdida rápida de partículas muy pequeñas o fibrosas, a través de los poros de la bolsa, durante las primeras horas de la incubación (Ørskov et al. 1980; Nocek, 1988), también por el alto contenido de cenizas en los SIP, ya que según Gojón et al. (1988), los minerales en algunos materiales con altos contenidos de cenizas pueden desaparecer hasta el $81 \%$, por difusión, a la hora cero de incubación ruminal. Espinoza et al. (2017) reportan degradabilidad superior al $78 \%$ en el tiempo 0 de incubación ruminal, en la fracción inorgánica de residuos agroindustriales.

La fracción "b" de la MS de los de SIP es similar a los reportados por Bravo et al. (2008), para residuos de cultivo de trigo y Contreras et al. (2019), para residuos de cosecha de maíz y superior a los valores registrados por Haile et al. (2017), para diferentes residuos de cultivos como maíz, cebada, sorgo, lentejas y trigo. Las características de degradabilidad para el FDA de los SIP muestran una mayor fracción "a", una menor fracción "b" y una baja tasa de degradación (kd $\left.\% \mathrm{~h}^{-1}\right)$.

La degradabilidad efectiva (DE) de la MS de los SIP, estimada con una tasa de pasaje de $5 \% \mathrm{~h}^{-1}$, encontrada en este trabajo, fue superior a los valores reportados por Chumpawadee et al. (2006) y
Haile et al. (2017), para diferentes residuos de cosecha, incluyendo leguminosas, como lentejas y Contreras et al. (2019), para residuos de cosecha de maíz y trigo.

Prueba de aceptación por los bovinos. Las novillas del primer estudio de aceptabilidad (Cuadrado latino 1) tuvieron un bajo consumo de los SIP ofrecidos (2,8\%); el consumo se incrementó al adicionar glicerol (41\%) o melaza (33\%) (Tabla 5).

Las novillas del segundo estudio de aceptabilidad (Cuadrado latino 2) consumieron cantidades similares del suplemento, cuando el SIP se mezcló con concentrado $(75 \%)$, que cuando a esta mezcla se le adicionó glicerol (73\%); sin embargo, estas novillas consumieron una mayor cantidad del suplemento $(89 \%)$, cuando a la mezcla de concentrado con SIP se le añadió melaza (Tabla 5).

Los SIP presentaron un bajo consumo cuando se suministraron solos, indicando una baja palatabilidad de este subproducto; el consumo se incrementó con el uso de glicerol o melaza. Los mayores consumos de SIP, se encontraron cuando se ofrecieron mezclados con el suplemento comercial y los palatabilizantes; sin embargo, autores, como Croy \& Rode (1988), reportaron que algunos

Tabla 5. Consumo de MS de los tratamientos utilizando SIP, suplemento comercial, glicerol y melaza, como parte de la ración de novillas Holstein (Cuadrados latinos).

\begin{tabular}{|c|l|c|c|c|}
\hline \multirow{2}{*}{$\begin{array}{c}\text { Cuadrados } \\
\text { latinos }\end{array}$} & \multicolumn{1}{|c|}{ Tratamiento } & $\begin{array}{c}\text { Oferta } \\
\text { tratamiento } \\
\text { kg MS/día }\end{array}$ & $\begin{array}{c}\text { CMS tratamiento } \\
\text { kg MS/día }\end{array}$ & $\begin{array}{c}\text { \% de CMS del } \\
\text { tratamiento } \\
\text { ofrecido }\end{array}$ \\
\hline \multirow{2}{*}{1} & T1. SIP & 1,07 & $0,030^{\mathrm{b}}$ & 2,8 \\
\cline { 2 - 5 } & T2. SIP + G & 1,28 & $0,53^{\mathrm{a}}$ & 41,4 \\
\cline { 2 - 5 } & T3. SIP + M & 1,28 & $0,42^{\mathrm{a}}$ & 32,8 \\
\hline \multirow{2}{*}{2} & T4. SIP + SC & 1,96 & $1,48^{\mathrm{b}}$ & 75,5 \\
\cline { 2 - 5 } & T5. SIP + SC + G & 2,17 & $1,60^{\mathrm{b}}$ & 73,7 \\
\cline { 2 - 5 } & T6. SIP + SC + M & 2,19 & $1,96^{\mathrm{a}}$ & 89,4 \\
\hline
\end{tabular}

SIP = Subproducto de la Industria del Papel; $\mathrm{G}=$ glicerol; $\mathrm{M}=$ Melaza; $\mathrm{SP}=$ suplemento comercial.

Promedio en la misma columna (para cada cuadrado latino) con letra distinta son estadísticamente diferentes $(\mathrm{p}<0,05)$. 
materiales, como residuos de pulpa de papel, pueden sustituir hasta el 36\% de una dieta a base de forraje, sin reducir el consumo, aunque encontraron un rechazo inicial en el consumo de los animales; después de 2 semanas la pulpa de papel fue consumida con facilidad. Coombe \& Briggs (1974) incorporaron residuos de papel en la dieta de ovinos, encontrando el consumo máximo de la ración, cuando el papel fue el $49 \%$ de la dieta; no encontraron cambio en el consumo al reducir la melaza en la dieta, pero registraron rechazo de la dieta por los animales, cuando incorporaron $84 \%$ de residuos de papel periódico. Moss et al. (1990) ofrecieron $4 \mathrm{~kg}$ de MS de pulpa de papel mezclada con heno de alfalfa en dietas para novillos, al tratar de incrementar en más de $6 \mathrm{~kg}$ de MS la pulpa de papel; hubo un rechazo de la ración. Mertens et al. (1971) evaluaron el consumo de tres niveles de inclusión de papel en la dieta de novillos (0,10 y 20\% de la ración), reportando diferencias significativas $(\mathrm{p}<0,05)$ y una respuesta inversa en el consumo (mayor consumo a menor inclusión de papel). Nishimura et al. (2019) evaluaron la inclusión de pulpa de papel $(12 \%)$ en la dieta de vacas lecheras, como remplazo del $50 \%$ del maíz de la ración y no encontraron diferencias significativas en el Consumo de Materia seca (CMS) respecto al tratamiento control. Maeda et al. (2019) incluyeron 12\% de pulpa de papel (Kraft) en dietas de novillos de engorde, sin evidenciar diferencias significativas en el CMS respecto al grupo control (concentrado y forraje), mientras que Izumi et al. (2019), al remplazar el ensilaje de maíz con la inclusión de pulpa de papel (Kraft) hasta un 57\% en dietas en vacas lecheras, encontró una reducción en el CMS, indicando que la inclusión de altos niveles de este tipo de materiales en la ración de los rumiantes, afecta el CMS.

Los SIP, al ser considerados residuos industriales no peligrosos, no requieren de tratamientos extras para su manejo y disposición final. Los resultados de los análisis de laboratorio y de digestibilidad, sugieren que, aunque este material tiene una baja calidad nutricional, puede tener un valor potencial en la alimentación de los rumiantes, como un componente para proporcionar MS, fibra y algunos minerales en las raciones de los animales; sin embargo, los altos contenidos de cenizas y, en especial, su contenido de cobre, plantea algunas limitaciones en la alimentación de algunos de los rumiantes.

Este tipo de material (SIP) presenta una baja palatabilidad y bajo consumo, cuando es suministrado solo a los animales; su consumo se incrementa cuando se incorpora glicerol o melaza, como palatabilizantes; sin embargo, los mayores consumos se encontraron cuando los SIP se mezclaron con un suplemento comercial y los palatabilizantes, principalmente melaza (Tabla 5). El integrar en la dieta de rumiantes cantidades de SIP aboga por la recirculación de nutrientes, evitando que estos materiales se vuelvan contaminantes del medio ambiente.

Recomendaciones. Los subproductos de la industria de papel (SIP) son un recurso viable como alternativa para suplir una fracción de la materia seca en los bovinos, cuando se presenta escases de oferta de alimento; sin embargo, se recomienda el uso de palatabilizantes para incrementar su consumo, debido a su baja aceptación, cuando es ofrecido como tal. Se debe tener en cuenta que compuestos, como el $\mathrm{Cu}$, que se encuentran en altos niveles en los SIP, pueden generar restricciones en su nivel de inclusión en dietas de especies, como los ovinos, que son poco tolerantes a altos niveles de este mineral.

Agradecimientos. A la empresa "Productos Familia Cajicá S.A.S", por el suministro de la materia prima, a la Gobernación de Cundinamarca, por incluir este trabajo en el proyecto de Innovación, Ciencia y Tecnología para productores de leche en la provincia de Ubaté Cundinamarca. Conflicto de intereses: El manuscrito fue preparado y revisado con la participación de todos los autores, quienes declaramos que no existe ningún conflicto de intereses que ponga en riesgo la validez de los resultados presentados.

\section{REFERENCIAS}

1. ANDI. 2019. Cámara de la Industria de Pulpa, Papel y Cartón. Informe de Sostenibilidad 2017. Primera edición. Radiografía de un sector comprometido con la sostenibilidad. Disponible desde internet en: http://www. andi.com.co/Uploads/Informe $\% 20 \mathrm{pulpa}^{2} 020 \mathrm{papel} \% 20$ y\%20carton\%20version2019 (con acceso 6/11/2019).

2. ARC. 1980. The Nutrient Requirements of Ruminant Livestock. Commonwealth Agricultural Búreaux. Slough, (England). $351 \mathrm{p}$.

3. ASSOCIATION OF OFFICIAL ANALYTICAL CHEMISTS, AOAC. 2006. Official Methods of Analysis of AOAC International. 18 ed. Animal Feed. (USA).

4. BAJPAI, P. 2015. Management of Pulp and Paper Mill Waste. Springer International Publishing. (Switzerland). 191p.

5. BERNAL, J. 2003. Pastos y forrajes tropicales. Manejo y producción. Cuarta edición. Ed. Ángel agro-Ideagro. (Colombia). 702p.

6. BHARGAVA, P.; ØRSKOV, E. 1987. Manual for the Use of Nylon Bag Technique in the Evaluation of Feedstuffs. Rowett Research Institute, Aberdeen. Scotland, (UK).

7. BRAVO, R.D.; ARELOVICH, H.M.; STORM, A.C.; MARTÍNEZ, M.F.; AMELA, M.I. 2008. Evaluación de métodos de amonificación mediante hidrólisis de urea sobre el valor nutritivo de paja de trigo. Rev. Argentina Producción Animal. (Argentina). 28(3):179-191.

8. CAVKA, A.; GUO, X.; TANG, S.; WINESTRAND, S.; JONSON, L.; HONG, F. 2013. Production of bacterial cellulose and enzyme from waste fiber sludge. Biotechnol. Biofuels (United Kingdom). Biotechnology for Biofuels. 6(1):25. https://doi.org/10.1186/1754-6834-6-25

9. CHUMPAWADEE, S.; SOMMART, K.; VONGPRALUB, T.; PATTARAJINDA, V. 2006. In Sacco degradation 
characteristics of crop residues and selected roughages in brahmanthai native crossbred steers. Kasetsart J. (Nat. Sci.) (Thailand). 40(1):204-214.

10. CONTRERAS, J.; MATOZ, M.; FELIPE, P.; CORDERO, F.; ESPINOZA, Y. 2019. Degradabilidad ruminal de forrajes y residuos de cosecha en bovinos Brown Swiss. Rev. Inv. Vet. Perú (Perú). 30(3):1117-1128.

11. COOMBE, J.; BRIGGS, A. 1974. Use of waste paper as a feedstuff for ruminants. Australian J. Experimental Agriculture and Animal Husbandry (Australia). 14:292-301.

12. CROY, D.; RODE, L. 1988. Nutritive value of pulp mill fiber waste for ruminants. Can. J. Anim. Sci. (Canadá). 68(2):461470 .

https://doi.org/10.4141/cjas88-052

13. ESPINOZA, I.; MEDINA, M.; BARRERA, A.; MONTENÉGRO, L.; SÁNCHEZ, A.; ROMERO, M.; GARCÍA, A. 2017. Composición bromatológica y degradabilidad ruminal in situ de residuos agroindustriales de maracuyá (Passiflora edulis) y plátano (Musa paradisiaca). Cienc Tecn UTEQ. (Ecuador) 10(2):63-67.

14. GOJÓN, B.; SIQUEIROS, D.; HERNÁNDEZ, H. 1988. In situ ruminal digestibility and degradability of Macrocystis pyrifera and Sargassum spp. in bovine livestock. Cien. Mar. (México). 24:463-481.

15. GÓMEZ, J.; CORREA, G.; BARAHONA, R. 2014. Evaluación del residuo del cultivo de Agaricus bisporus como alimento de vacas lecheras en lactancia media. Rev. Fac. Nal. Agr. Medellín (Colombia). 67(2):7331-7343. https://doi.org/10.15446/rfnam.v67n2.44175

16. GOSELINK, R.; JONGBLOED, A. 2012. Zinc and copper in dairy cattle feeding. Wageningen UR livestock research partner in livestock innovations. report 519 (Netherland). $31 \mathrm{p}$.

17. HAILE, E.; GICHEHA, M.; NJONGE, F.; ASGEDOM, G. 2017. Determining Nutritive Value of Cereal Crop Residues and Lentil (Lens esculenta) Straw for Ruminants. Open J. Animal Sciences. 7:19-29. https://doi.org/10.4236/ojas.2017.71003

18. HOWARD, R.; ABOTSI, E.; JANSEN VAN RENSBURG, E.; HOWARD, S. 2003. Lignocellulose biotechnology: tissues of bioconversion and enzyme production. African J. Biotechnology. (Nigeria). 2(12):602-619.

19. IZUMI, K.; TAMURA, T.; FUJII, R.; NAKATSUJI, H.; MORITA, S. 2019. Effects of substituting kraft pulp with corn silage on dry matter intake, ruminal mat formation, rumen fermentation, and rumination activity in non- lactating cows. Anim Sci J. (United Kingdom). 90 (2):2052013.

https://doi.org/10.1111/asj.13149

20. KUJALA, A. 2012. Papermaking sludge and possibilities of utilization as material. Bachelor Seminar of Environmental Technology. Lappeenranta University of Technology. Faculty of Technology. (Finland). 68p. Disponible desde internet en:

http:/ /www.doria.fi/bitstream/handle/10024/73980/ Kujala $\% 20$ Asta_Kandidaatinty $\%$ C3\%B6.pdf?sequence $=1$ (con acceso 15/11/2016).

21 MAEDA, Y.; NISHIMURA, K.; KUROSU, K.; MIZUGUCHI, H.; SATO, S.; TERADA, F.; KUSHIBIKI, S. 2019. Effect of feeding wood kraft pulp on the growth performance, feed digestibility, blood components, and rumen fermentation in Japanese Black fattening steers. Anim Sci J. (United Kingdom). 90(4):523-532. https://doi.org/10.1111/asj.13182

22. MAHESH, M.; MOHINI, M. 2013. Biological treatment of crop residues for ruminant feeding: A review. Afr. J. Biotechnol. (Nigeria). 12(27):4221-4231. https://doi.org/10.5897/AJB2012.2940

23. MARTÍNEZ, R.; MARTÍNEZ, N.; MARTÍNEZ, M. 2011. Diseño de experimentos en ciencias agropecuarias y biológicas con SAS, SPSS, R y Statistix. Tomo I. Primera edición. Fondo Nacional Universitario. (Colombia). 399p.

24. MÉNDEZ, A.; FIDALGO, J.; GUERRERO, F.; GASCÓ, G. 2009. Characterization and pyrolysis behavior of different paper mill waste materials. J. Anal. Appl. Pyrolysis (Netherlands). 86:66-73.

https://doi.org/10.1016/j.jaap.2009.04.004

25. MERTENS, D.; CAMPBELL, J.; MARTZ, A. 1971. Lactational and ruminal response of dairy cows to ten and twenty percent dietary newspaper. J. Dairy Science (USA). 54(5):667-672.

https://doi.org/10.3168/jds.S0022-0302(71)85904-0

26. MINISTERIO DE AMBIENTE, VIVIENDA Y DESARROLLO TERRITORIAL. 2005. Decreto número (4741) 30 de diciembre de 2005. "Por el cual se reglamenta parcialmente la prevención y manejó de los residuos o desechos peligrosos generados en el marco de la gestión integral". Disponible desde internet en: https:/ / diario-oficial.vlex.comco/vid/decreto-474143232025?_ga $=2.49572576 .716973682 .1573274357$ 1623162950.1573274357 (con acceso 05/11/2019).

27. MINISTERIO DE VIVIENDA CIUDAD Y TERRITORIO. 2014. Decreto número (1287) 10 de Julio de 2014. "Por el cual se establecen criterios para el uso de los biosólidos 
generados en plantas de tratamiento de aguas residuales municipales". Disponible desde internet en: http://www.minvivienda.gov.co/Decretos $\% 20$ Vivienda/1287\%20-\%202014.pdf (con acceso 05/11/2019).

28. MONTE, M.; FUENTE, E.; BLANCO, A.; NEGRO, C. 2009. Waste management from pulp and paper production in the European Union. Waste Manag. (United Kingdom). 29(1):293-308.

https://doi.org/10.1016/j.wasman.2008.02.002

29. MOSS, J.; EHRLICH, E.; BUCHANAN, K.; MARTIN, P. 1990. Evaluation of recycled paper pulp residue as a feed of cattle. Proc. Aust, Soc. Anim. Prod. (Australia). 18:316:319.

30. NATIONAL RESEARCH COUNCIL, NRC. 2001. Nutrient Requirements of Dairy Cattle: Seventh Revised Edition. National Academy Press. Washington D.C.

31. NISHIMURA, K.; KUROSU, K.; TERADA, F.; MIZUGUCHI, H.; SATO, S.; KUSHIBIKI, S. 2019. Effect of wood kraft pulp feed on digestibility, ruminal characteristics, and milk production performance in lactating dairy cows. Anim Sci J. (United Kingdom). 90(2):189-195.

https://doi.org/10.1111/asj.13131

32. NOCEK, J. 1988. In situ and other methods to estimate Ruminal Protein and Energy Digestibility: a review. J. Dairy Sci. (USA) 71(8):20512069.

https://doi.org/10.3168/jds.S0022-0302(88)79781-7

33. ØRSKOV, E. 2000. The in situ technique for the estimation of forage degradability in ruminants. In: GIVEN, D.I.; OWENS, E.; AXFORD, R.F.E. and Omed, H.D (Eds.). Forage evaluation in ruminant nutrition. Wallingford: $\mathrm{CAB}$ International. p.175-188.
34. ØRSKOV, E.; DEB HOVELL, F.; MOULD, F. 1980. The use of the nylon bag technique for the evaluation of feedstuffs. Trop. Anim. Prod. (Netherlands). 5(3):195-213.

35. ØRSKOV, E.; MCDONALD, I. 1979. The estimation of protein degradation in the rumen from incubation measurements weighted according to rate of passage. J. Agric. Sci. Camb. (USA). 92:499-503.

https://doi.org/10.1017/S0021859600063048

36. TILLEY, J.; TERRY, R. 1963. A two stage technique for in vitro digestion of forage crops. J. Br. Grassl. Soc. (Australia). 18:104-111

37. VAN SOEST, P.J.; ROBERTSON, J.B.; LEWIS, B.A. 1991. Methods for dietary fiber, neutral detergent fiber, and nonstarch polysaccharides in relation to Animal nutrition. J. Dairy Sci. (USA). 74:3583-3597. https://doi.org/10.3168/jds.S0022-0302(91)78551-2

38. VAN SOEST, P.J. 1994 Nutritional Ecology of the Ruminant. 2nd ed. Cornell University Press, Ithaca, NY. 476p.

39. VILLAS-BÔAS, S.G.; ESPOSITO, E.; MITCHELL, D. 2002. Microbial conversion of lignocellulosic residues for production of animal feeds. Animal Feed Science and Technology (Netherlands). 98(1-2):1-12. https://doi.org/10.1016/s0377-8401(02)00017-2 